\title{
Independent component analysis of geoelectric field data in the northern Nagano, Japan
}

\author{
By Yoshiaki OrIhara, ${ }^{* 1}$ Masashi Kamogawa, ${ }^{* 2, \dagger}$ Toshiyasu NAGaO ${ }^{* 1}$ and Seiya UyedA ${ }^{* 3}$
}

\author{
(Contributed by Seiya UYEDA, M.J.A.)
}

\begin{abstract}
Simultaneous anomalous change of geoelectric field was observed on January 17, 1999 at three far-distant stations in Nagano Prefecture, Japan. Eleven days after the anomalous change, an earthquake swarm with a M4.8 main shock started at a location within the triangle formed by the three stations. This change was rectangular in shape like the VAN-type anomalous change in Greece. The anomaly could be detected because it occurred at midnight when the noise level was low. In fact, there were a few M4-class earthquakes in the area for which no preceding anomalous electric changes were observed, perhaps because the changes occurred during the noisy daytime. In this paper, we examine if it is possible to extract the signal with the size of the anomalous change on January 17, 1999 from the noisy daytime data by Independent Component Analysis. For this purpose, a simulated noise was embedded in the real daytime data which were highly influenced by not only artificial noise but also by geomagnetic disturbances and rainfall. Even when the influence of geomagnetic disturbance or rainfall was large, the anomalous change was clearly recognized at least at two stations in all cases.
\end{abstract}

Keywords: geoelectric field, Independent Component Analysis (ICA), earthquake precursor

\section{Introduction}

Anomalous changes of geoelectric field, termed SES (seismic electric signals), have been observed before earthquakes since 1980s in Greece. ${ }^{1), 2)}$ The Greek authors discriminate SES and other changes caused by geomagnetic disturbances, instability of electrodes, artificial noises and so on by using short and long measuring dipoles and applying certain criteria summarized in the reference. ${ }^{3)}$ This observation method, called VAN method, has been used for earthquake prediction in Greece. ${ }^{4)}$ The duration of SES ranges from a few minutes to several hours and the amplitude is of the order of $10^{-5} \mathrm{~V} / \mathrm{m}$. The SES usually appears within several weeks before the main shock.

In Japan, anomalous geoelectric field changes that might be SES were observed before several earthquakes.5)-8) On January 17, 1999, an anomalous

*1 Earthquake Prediction Research Center, Tokai University, Shizuoka, Japan.

*2 Department of Physics, Tokyo Gakugei University, Tokyo, Japan.

*3 Japan Academy, Tokyo, Japan.

$\dagger$ Correspondence should be addressed: M. Kamogawa, 4-1-1 Nukuikitamachi, Koganei, Tokyo 184-8501, Japan (e-mail: kamogawa@u-gakugei.ac.jp). change with a rectangular shape and 27-minute duration was observed simultaneously at three fardistant stations located at Hakuba (HKB), Matsushiro (MTS), and Outaki (OTA), in Nagano Prefecture, central Japan (Fig. 1). The amplitude of the signal was $1.9,2.3$, and $1.2 \times 10^{-5} \mathrm{~V} / \mathrm{m}$ at $\mathrm{HKB}$, MTS, and OTA, respectively. Since an earthquake swarm with a M4.7 main shock started 11 days after the anomalous change in the midst of the triangle formed by the three stations, this change was considered to be the SES of that main shock. ${ }^{9)}$

In Japan, it is difficult to detect SES because of high noise due to leakage currents from DC driven trains and other artificial sources, besides natural changes such as rainfall, lightning, and induction of geomagnetic field variations and ocean-earth tides. The DC leakage from trains, in particular, produces so large amplitude of geoelectric field changes that can even be utilized as a current source of resistivity surveys for areas with sizes of square of a few tens of kms. ${ }^{10)}$ The anomalous change observed at the three far-distant stations in Nagano Prefecture was detected because it occurred at midnight when no electric train was running. Therefore, Yamaguchi et al. $\left.{ }^{9}\right)$ tried to extract such a change from daytime data by 


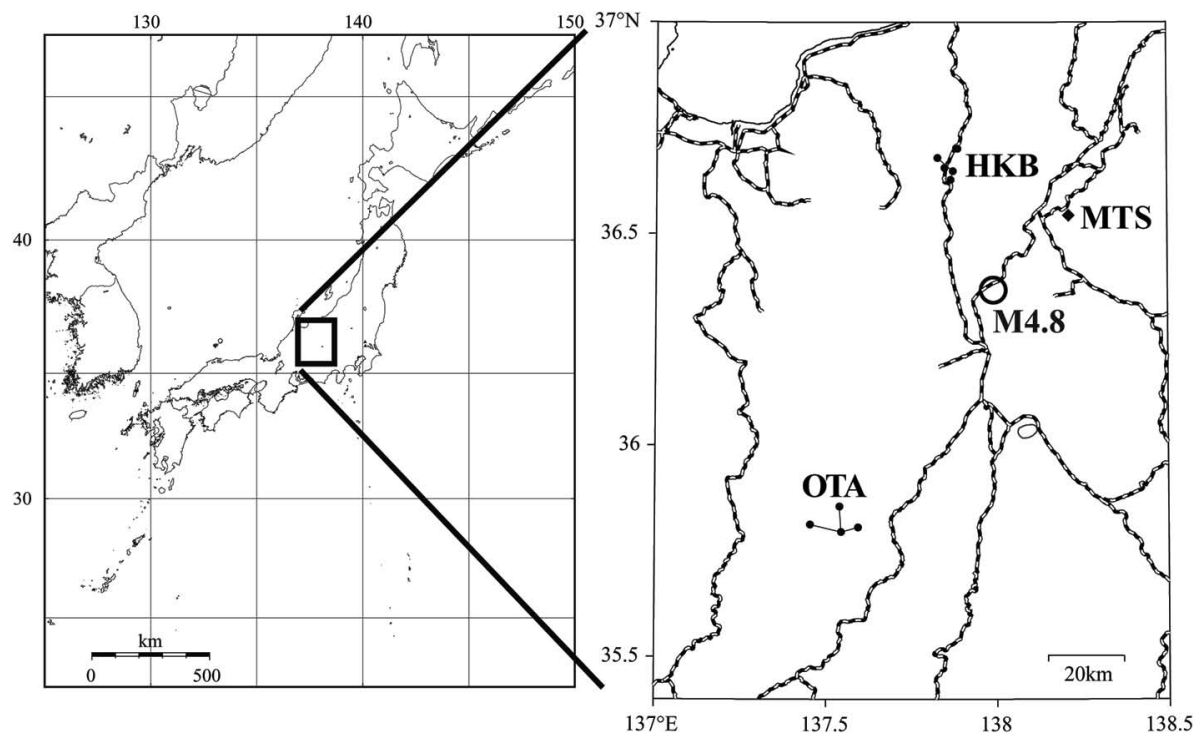

Fig. 1. Three stations where the signals treated in this study were observed. In the right panel, circle shows the epicenter of the M4.8 earthquake on January 28, 1999 and dotted lines denote railroad tracks.

using the Particle Motion Diagram (PMD) method. In the PMD method, the time-series of variation in the direction perpendicular to that of the predominant noise in Lissajous-type figure is obtained. If the target variation has component perpendicular to that of predominant noise, the time-series in the latter direction should suffer from less noise. However, no anomalous change was detected when this method was applied to the whole observed timeseries from October 1 of 1998 to June 30 of 1999. The separation of signals was also tried by using neural network on a MTS (Matsushiro) data with train noise in which rectangular change with similar amplitude was artificially embedded. ${ }^{11}$ However, the computational cost of neural network noise reduction is so large even for the time-series of one-day data. So, the method is not easily applied to long-term timeseries. On the other hand, it was suggested that Independent Component Analysis (ICA) was a better solution. ${ }^{12), 13)}$

In this paper, we investigate the possibility of extraction of the signal similar to the rectangular change of January 17, 1999 embedded in selected daytime data observed at the three stations by using Independent Component Analysis (ICA).

\section{Independent component analysis}

ICA is a computational method to extract source signals from observed mixture data under the assump- tion that the source signals are linearly added. Source signals $\mathbf{s}(t)$ consists of signals generated from $n$ sources which are mutually independent and the signals $\mathbf{x}(t)$ observed at $n$ points are expressed by

$$
\begin{aligned}
\mathbf{s}(t) & =\left(s_{1}(t), s_{2}(t), \cdots, s_{n}(t)\right)^{\mathrm{T}} \\
\mathbf{x}(t) & =\left(x_{1}(t), x_{2}(t), \cdots, x_{n}(t)\right)^{\mathrm{T}} .
\end{aligned}
$$

The relationship between $\mathbf{s}(t)$ and $\mathbf{x}(t)$ is written by

$$
\mathbf{x}(t)=\mathbf{A} \mathbf{s}(t)
$$

where $\mathbf{A}$ is termed mixing matrix. When inverse matrix $\mathbf{W}$ of $\mathbf{A}$ can be obtained, the independent signals $\mathbf{y}(t)$ are obtained from $\mathbf{x}(t)$ by

$$
\mathbf{y}(t)=\mathbf{W} \mathbf{x}(t)
$$

where $\mathbf{y}(t)=\left(y_{1}(t), y_{2}(t), \ldots, y_{n}(t)\right)^{\mathrm{T}}$. If the estimation of $\mathbf{W}$ is correct, $\mathbf{y}(t)$ is expected to correspond to original source signals $\mathbf{s}(t)$.

To estimate $\mathbf{W}$ based on equation [4], many algorithms have been proposed. ${ }^{14)-16)}$ In this paper, we use FastICA algorithm. ${ }^{15), 17)}$ FastICA is often used for the blind signal separation and the bioinstrumentation signal analysis, etc., and high speed calculation is its advantage. The results obtained by this algorithm are generally appropriate. ${ }^{17)}$

\section{Signal separation by ICA}

Anomalous rectangular change was observed at three far-distant stations (HKB, MTS, OTA) in Na- 
(a)

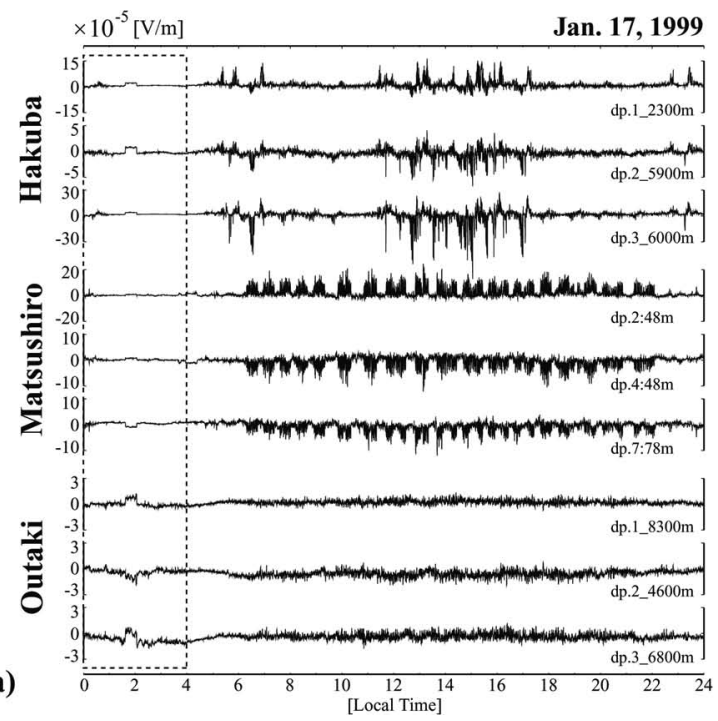

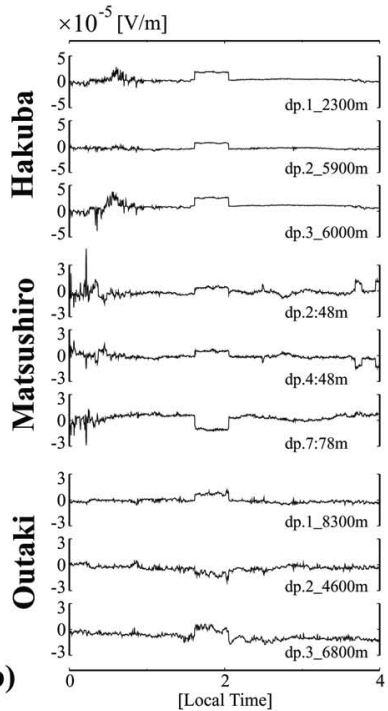

Fig. 2. Time-series of geoelectric field at three stations: Hakuba (HKB), Matsushiro (MTS), and Outaki (OTA). An anomalous rectangle change was observed at midnight of January 17, 1999. (a) One-day data. (b) Midnight data. The sampling rate of the observation was 10 seconds at the three stations.

gano Prefecture at midnight (Figs. 1, 2). According to Koganeyama et al., ${ }^{12)}$ rectangular change signals observed at three MTS dipoles and train noise were separately extracted by ICA from a daytime data into which the signals were artificially embedded. In this paper, we applied the same procedure to the data observed at all three stations. Six daytime data were chosen from Oct. 1, 1998 to Dec. 31, 1999. Some of them were affected by not only train noises but also by different levels of geomagnetic disturbance and rainfall (Table 1). Figure 3 shows dipole configurations at the three stations. There were only three long dipoles at HKB and OTA. Station MTS had 8 short dipoles, but optimally oriented three of them, i.e., dipole 2, 4, and 7 were selected (Fig. 3). The data length of this analysis was 20 hours (from 04:00 to 24:00 LT, termed daytime), because the intensity of the artificial noise mainly caused by train operation was large during this time interval. The rectangular change signals as observed on January 17, 1999 (duration $1600 \mathrm{sec}$ ) were embedded in the selected data (See Table 2). The embedding of rectangular change was made at three times on each testing day on each station data: 1) 08:00 to $08: 26: 30,2$ ) $13: 00$ to $13: 26: 30$, and 3$) 18: 00$ to $18: 26: 30$. One example of embedded data is shown in Figs. 4a,b. It is difficult to recognize the rectangular embedded signals even when we know the embedding time. After
ICA is applied to the embedded data, it is clear to recognize the rectangular shape, the train noise, and the residual components as shown in Fig. 4b. Since the absolute amplitude of the separated time-series is lost in ICA, ${ }^{17)}$ we followed the amplitude reproduction procedure developed by Koganeyama et al. ${ }^{12)}$ Figures $4 \mathrm{~b}$ show the reproduced amplitude by using this procedure. Since the reproduction procedure is incomplete, the reproduced amplitude is not exactly the same as the original one as shown in Figs. 4a and $4 \mathrm{~b}$. Table 1 shows the results of 18 combinations of the three embedding times on the six testing days at the three stations. Figure 5 shows some excerpts from Table 1; namely six examples of three independent components of dipole 1 at HKB, dipole 2 at MTS, and dipole 1 at OTA after the ICA procedure. The polarity is sometimes reversed as shown in $5 f$.

Results and discussion. In Table 1, the signal detectability is categorized as detectable (O), barely detectable $(\square)$, and undetectable $(-)$. The criteria are as follows: 1) Detectable: Rectangular change is clearly recognized in one or two components. 2) Barely detectable: Rectangular change is recognized only with the help of simultaneous records at other station. 3) Undetectable: Recognize rectangular change is unrecognizable even with simultaneous records at other station. Evaluation of the overall detectability at each station was made, by 
Table 1. Rainfall data recorded by JMA near the three stations (HKB, MTS, and OTA) and Ap index. Anomalous rectangle signals were embedded in 8:00, 13:00, and 18:00. Detectability of embedded anomalous rectangle signals after ICA process at each station is shown by marks "O" (detectable), " $\square$ " (barely detectable), and "_" (undetectable)

\begin{tabular}{|c|c|c|c|c|c|c|c|}
\hline & Date & $\begin{array}{c}\text { Rainfall }[\mathrm{mm}] \\
\text { (HKB, MTS, and OTA) }\end{array}$ & Ap index & Embedded time & HKB & MTS & OTA \\
\hline \multirow{3}{*}{1} & & & & 08:00 & $\square$ & O & O \\
\hline & Feb. 16, 1999 & $0,0,0$ & 4 & $13: 00$ & $\square$ & O & O \\
\hline & & & & 18:00 & O & O & O \\
\hline \multirow{3}{*}{2} & & & & 08:00 & - & O & O \\
\hline & Nov. 8, 1998 & $0,0,0$ & 66 & $13: 00$ & - & O & O \\
\hline & & & & 18:00 & - & O & 0 \\
\hline \multirow{3}{*}{3} & & & & 08:00 & $\square$ & 0 & O \\
\hline & Jul. 18, 1999 & $13,11,6$ & 4 & $13: 00$ & $\square$ & O & O \\
\hline & & & & 18:00 & $\square$ & 0 & $\square$ \\
\hline \multirow{3}{*}{4} & & & & 08:00 & $\square$ & O & $\square$ \\
\hline & Sep. 22, 1999 & $52,31,33$ & 50 & $13: 00$ & $\square$ & O & $\square$ \\
\hline & & & & 18:00 & $\square$ & O & $\square$ \\
\hline \multirow{3}{*}{5} & & & & 08:00 & $\square$ & O & O \\
\hline & May 3, 1999 & $0,0,0$ & 8 & $13: 00$ & O & O & O \\
\hline & & & & 18:00 & O & O & O \\
\hline \multirow{3}{*}{6} & & & & 08:00 & $\square$ & O & O \\
\hline & Aug. 1, 1999 & $0,0,0$ & 8 & $13: 00$ & O & O & O \\
\hline & & & & $18: 00$ & $\square$ & O & O \\
\hline
\end{tabular}

(a)

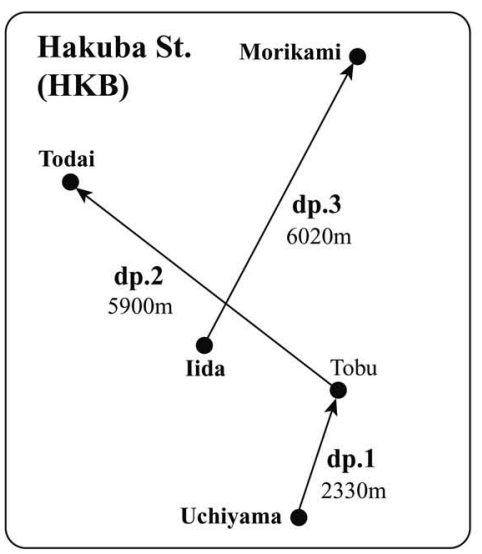

(b)

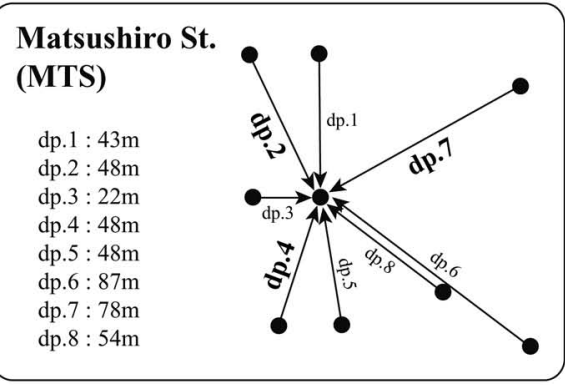

(c)

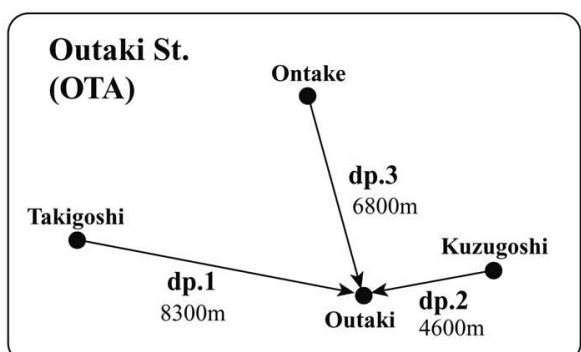

Fig. 3. Configuration map of electric dipoles at the three geoelectric field stations. (a) Long dipoles at Hakuba. (b) Short dipoles at Matsushiro. (c) Long dipoles at Outaki. 
Table 2. Amplitude of the anomalous rectangle signals observed at three dipoles of the three stations. Unit is $\mu \mathrm{V} / \mathrm{m}$

\begin{tabular}{lrlrlr}
\hline \multicolumn{2}{l}{ Hakuba (HKB) } & \multicolumn{2}{c}{ Matsushiro (MTS) } & \multicolumn{2}{c}{ Outaki (OTA) } \\
\hline dp.1 & +15 & dp.2 & +12 & dp.1 & +7 \\
dp.2 & +12 & dp.4 & +12 & dp.2 & -6 \\
dp.3 & +16 & dp.7 & -20 & dp.3 & +12 \\
\hline
\end{tabular}

giving two, one, and zero point to "O", " $\square$ ", and "_" respectively. The score is 36 at MTS, 32 at OTA, and 19 at HKB. Only at station MTS, the rectangle changes were extracted under all the conditions of rainfall and geomagnetic disturbance. In the total of 18 cases studied, simultaneous recovery of the embedded changes at three stations was realized in 15 cases. There were three cases where the simultaneous recovery was attained at two stations. Thus, in all the cases, the simultaneous recovery was found at least at two stations.

We focus on the difference of detectability between the stations. In general, most of DC electric current supplied from an electric-power substation to the train returns from the train to the substation through the metallic rail, while the rest of them returns though the earth's ground as a leakage current. The source dipole of leakage current between the sub- station and the train varies when the train moves. ${ }^{18)}$ MTS is located at $2 \mathrm{~km}$ south of Nagano Electric Railway, OTA at $10 \mathrm{~km}$ west of Japan Railways (JR) Chuo Main Line and $20 \mathrm{~km}$ east of JR Takayama Main Line, and the two long dipoles 2 and 3 of HKB (Fig. 3a) are intersected by JR Ohito Line. Influence of the train was expected to be less at OTA than at MTS and HKB because OTA is located far from the railway. At MTS, heavy leakage current from trains was observed according to Yamaguchi et al.9) Although the train source signal moves, it may be regarded as a fixed source at MTS, because the distance between MTS and the railway is $2 \mathrm{~km}$, which is larger than the short dipole length in operation at MTS (Fig. 3b). According to Ishikawa et al., ${ }^{19)}$ there is a clear correlation between variations of observed geoelectric field at HKB and leakage current from the electric substation for the train. As mentioned above, at HKB, long measuring dipoles intersect Ohito Line. Therefore, the configuration of train source dipole and the measuring long dipoles at HKB drastically varies when the train moves, which differs from the case of MTS. Less detectability was resulted at HKB than at MTS and OTA, because fastICA algorithm is designed for fixed signal sources.

Detectability at OTA seems to be affected by rainfall more than at $\mathrm{HKB}$ and MTS. The reason for this difference is not clear at this moment. Large

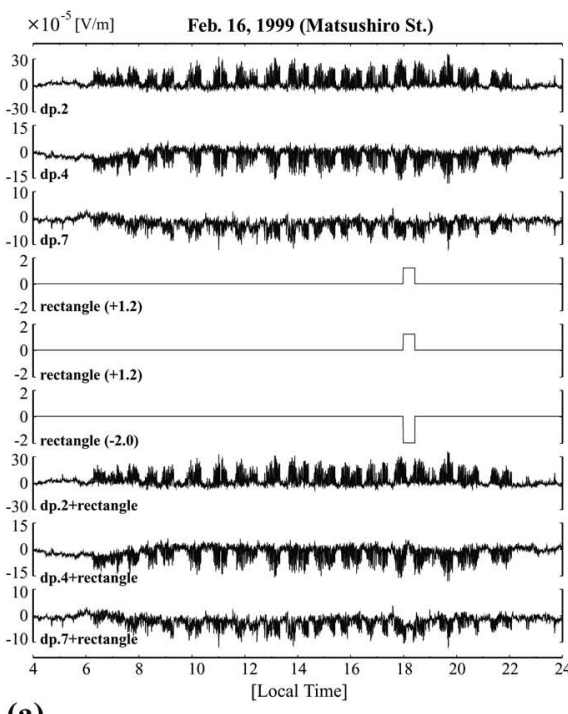

(a)

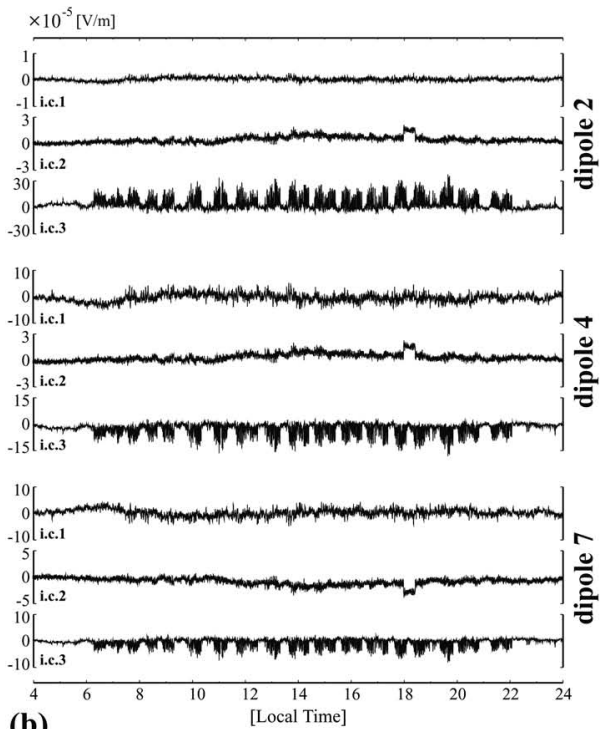

(b)

Fig. 4. (a) Top three panels: original data recorded on Feb. 16, 1999 at dipoles 2, 4, and 7 of MTS station. Middle three panels: Actually observed rectangle signals at respective dipoles to be embedded. Bottom three panels: data containing embedded rectangle signals. (b) Three independent components of the three dipoles separated after the employed ICA. ${ }^{11), 16)}$ 

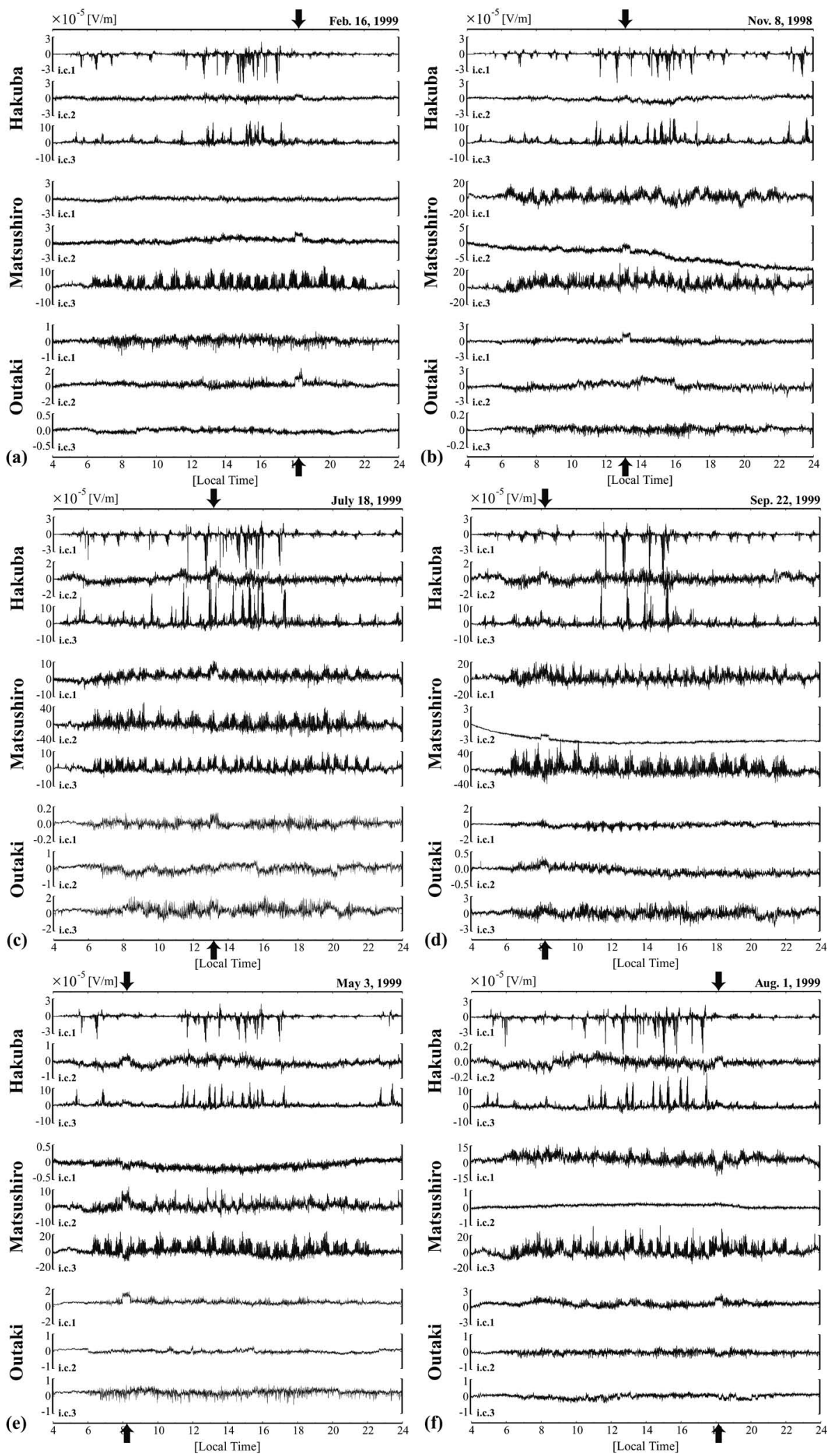

Fig. 5. Three independent components of dp.1 at Hakuba, dp.2 at Matsushiro, and dp.1 at Outaki. Arrows indicate the embedding time of the anomalous rectangle signals observed at respective dipoles. (a) Case of no rainfall and less geomagnetic activity on February 16, 1999. Signal appeared in one independent component of three stations. (b) Case of no rainfall and large 
(Fig. 5. continued)

geomagnetic activity on November 8, 1998. Signal appeared in two independent components of Matsushiro station. (c) Case of rainfall and less geomagnetic activity on July 18, 1999. Signal cannot be recognized in Hakuba data, but can be confirmed in Matsushiro data. Signal was barely recognizable in Outaki data. (d) Case of rainfall and large geomagnetic activity on September 22, 1999. At Matsushiro, clear rectangle change appeared in second independent component which also showed a slow drift. Simultaneous change in Hakuba and Outaki can barely be recognized in the second and third and second components, respectively. (e) Case of May 3, 1999; one of the normal days (no rainfall and moderate geomagnetic activity). Although the rectangle signal at Hakuba was small and unclear, the signal at the others is clear. (f) Another normal day on August 1, 1999 .

geomagnetic activity seems to affect the separation capability at MTS because the embedded signal appeared in more than one independent components (Fig. 5b, d), while no such change was observed at HKB and OTA. According to Yamaguchi et al. ${ }^{9)}$ apparent coming direction of the anomalous signal of January 17, 1999 was west at MTS while that at HKB and OTA was south and north respectively. It is known that, in general, large east - west component of telluric current is induced during active geomagnetic variations. Since the apparent coming direction of the anomalous signal at MTS was west, the geomagnetic activity affected the detectability only at MTS.

PMD, using two components of observed data, showed that separation between train source and anomalous change was made at MTS and HKB, but not at OTA, because the dominant direction in Lissajour-type figure did not appear at OTA. As mentioned above, using three dipole data at OTA, components of artificial noise and anomalous change were well-separated by ICA. It is, thus, re-confirmed that ICA is a better method to separate components in geoelectric field variations than PMD.

\section{Conclusion}

We discussed the possibility of separating components of geoelectric field variations from noisy daytime data at the three stations in Nagano Prefecture by using ICA. From the ICA analysis, the simultaneous change could be recognized in the daytime data at least at two stations even under the rainfall and geomagnetic activity conditions. The reason why the separation was more difficult at HKB than MTS and OTA was the varying configuration relationship between the measuring dipoles and the leakage current source dipole from the trains.

\section{Acknowledgements}

The geoelectric field measurement at Matsushiro was carried out as a part of the joint research of Seis- mological and Volcanological Department of the Japan Meteorological Agency (JMA) and International Research Program on Earthquake Prediction of the Institute of Physical and Chemical Research (RIKEN). We thank Dr. I. Takahashi, Mr. Y. Noda, Mr. T. Yamaguchi for operating the long-term observation. Authors are also grateful to Professors K. Yamazaki (Tokyo Gakugei University) and A. Mori (Obirin University) for their supports and useful comments. This research was partially supported by the Ministry of Education, Culture, Sports, Science and Technology, Grant-in-Aid for Young Scientists (B), No. 21710180, 2009 (M. K.), and Scientific Research (C), No. 20510171, 2008 (S. U. and M. K.), Ito Science Foundation, 2007 (M. K.) and Grant-inAid of Fukada Geological Institute, 2009 (Y. O. and M. K.).

\section{References}

1) Varotsos, P. and Alexopoulos, K. (1984) Physical properties of the variations of the electric field of the earth preceding earthquakes, I. Tectonophysics 110, 73-98.

2) Varotsos, P. and Alexopoulos, K. (1984) Physical properties of the variations of the electric field of the earth preceding earthquakes, II, Determination of epicenter and magnitude. Tectonophysics $\mathbf{1 1 0}$ 99-125.

3) Varotsos, P. and Lazaridou, M. (1991) Latest aspects of earthquake prediction in Greece based on Seismic Electric Signals, Tectonophysics 188, 321-347.

4) Uyeda, S. and Kamogawa, M. (2008) The prediction of two large earthquakes in Greece, Eos 89, doi: 10.1029/2008EO390002.

5) Nagao, T., Uyeda, S., Asai, Y. and Kono, Y. (1996) Anomalous changes in geoelectric potential predicting four earthquakes in Japan, A critical review of VAN earthquake prediction from seismic electrical signals, World Scientific, London, pp. 292300 .

6) Uyeda, S., Nagao, T., Orihara, Y., Yamaguchi, T. and Takahashi, I. (2000) Geoelectric potential changes: Possible precursors to earthquakes in Japan. Proc. Nat. Acad. Sci. U.S.A. 97, 4561-4566.

7) Orihara, Y., Noda, Y., Nagao, T. and Uyeda, S. (2002) A possible case of SES selectivity at Kozushima Island, Japan. J. Geodynamics 33, 425-432. 
8) Uyeda, S., Hayakawa, M., Nagao, T., Molchanov, O., Hattori, K., Orihara, Y. et al. (2002) Electric and Magnetic phenomena observed before the volcanoseismic activity 2000 in the Izu islands region, Japan. Proc. Nat. Acad. Sci. U.S.A. 99, 7352-7355.

9) Yamaguchi, T., Noda, Y., Takahashi, I., Orihara, Y., Hattori, K., Kawabata, K., Nagao, T. and Uyeda, S. (2000) A regionally observed simultaneous preseismic geoelectric potential change in Nagano Prefecture, Japan. Bull. Inst. Oceanic Res. \& Develop., Tokai Univ. 21, 11-22 (in Japanese).

10) Tanbo, T., Nagao, T. and Sakai, H. (2002) Geoelectric study using the leak current from railroad as a signal, Application to Ohtawa station, Gifu Prefecture, Japan. Proc. Jpn. Acad., Ser. B 78, 179-184.

11) Koganeyama, M., Sugita. M., Nagao, T. and Joe, K. (2000) Removing train noise from telluric current data by neural networks for automatic short-term earthquake prediction in Japan. IPSJ SIG Notes 60, 29-32.

12) Koganeyama, M., Shouno, H., Nagao, T. and Joe, K. (2002) Separation of train noise and seismic electric signals in telluric current data by ICA. Trans. Inf. Process. Soc. Japan 43, 92-104 (in Japanese).

13) Ishikawa, C., Watanabe, C., Nagao, T. and Joe, K. (2005) Extracting seismic electronic signals from the telluric current data for the Niigata Chuetsu Earthquake by ICA. Proc. Int. Conf. on Parallel and Distributed Process. Tech. Appl. II, 488-494.

14) Cardoso, J. F. and Laheld, B. (1996) Equivariant adaptive source separation. IEEE Trans. Signal Process. 44, 3017-3030.

15) Hyvarinen, A. and Oja, E. (1997) A fast fixed-point algorithm for independent component analysis. Neural Comp. 9, 1483-1492.

16) Cichocki, A. and Amari, S. (2002) Adaptive Blind Signal and Image Processing. John Wiley \& Sons, Inc., New York, pp. 1-554.

17) Hyvarinen, A., Karhunen, J. and Oja, E. (2001) Independent Component Analysis. John Wiley \& Sons, Inc., New York, pp. 1-481.

18) Yanagihara, K. (1977) Magnetic field disturbance produced by electric railway. Mem. Kakioka Magnetic Observatory, Supplementary 7, 17-36.

19) Ishikawa, H., Hattori, K., Takahashi, I., Noda, Y. Nagao, T. and Isezaki, N. (2007) Effect of noise from DC-driven trains to geoelectrical potential difference and its reduction in Hakuba area, Japan. IEEJ Trans. 127, 41-47.

(Received Sept. 29, 2009; accepted Oct. 5, 2009) 